\title{
How do Chemical Cleaning Agents Act on Polyamide Nanofiltration Membrane and Fouling Layer?
}

Jiachen Huang, ${ }^{\dagger,} \S$ Jianquan Luo, ${ }^{*}, \dagger, \S$ Xiangrong Chen, ${ }^{\dagger}, \S$ Shichao Feng, ${ }^{\dagger, \S}$ Yinhua

$$
\mathrm{Wan}^{*}, \dagger, \S
$$

†State Key Laboratory of Biochemical Engineering, Institute of Process Engineering,

Chinese Academy of Sciences, Beijing 100190, PR China

§School of Chemical Engineering, University of Chinese Academy of Sciences, Beijing 100049, PR China

*Corresponding author (Tel: +010-62543301, email: jqluo@ipe.ac.cn, yhwan@ipe.ac.cn) 
Table S1. Ingredients, properties and functions of commercially available cleaning agents.

\begin{tabular}{|c|c|c|c|}
\hline Manufacturer & Trade name & Components/properties & Target foulants \\
\hline \multicolumn{4}{|c|}{ Commercial cleaning agents designed for inorganic fouling } \\
\hline \multirow{3}{*}{ Ecolab } & $\begin{array}{c}\text { PermaClean } \\
33\end{array}$ & $\mathrm{pH} 12-13$ & $\begin{array}{c}\text { Acid-insoluble sulfates of } \\
\text { calcium, barium and strontium as } \\
\text { well as calcium fluoride }\end{array}$ \\
\hline & $\begin{array}{c}\text { PermaClean } \\
67\end{array}$ & $\begin{array}{l}\text { A neutral } \mathrm{pH} \text { cleaner including an } \\
\text { anionic surfactant }\end{array}$ & $\begin{array}{l}\text { Inorganic colloids and other } \\
\text { organic matter }\end{array}$ \\
\hline & Ultrasil 73 & $\begin{array}{l}\text { An acidic surfactant-based liquid } \\
\text { cleaner }\end{array}$ & $\begin{array}{c}\text { Inorganic soil as well as fat and } \\
\text { mineral oil }\end{array}$ \\
\hline BWA & Clocon MC3 & $\begin{array}{l}\text { Mixture of organic acids, detergent } \\
\text { builders and chelating agents }\end{array}$ & $\begin{array}{l}\text { Metal hydroxides, calcium } \\
\text { carbonate and other similar } \\
\text { scaling }\end{array}$ \\
\hline Suez (GE) & $\begin{array}{l}\text { MemChem } \\
\text { MCT201 }\end{array}$ & $\begin{array}{c}\text { A low } \mathrm{pH} \text { solid formulation } \\
\text { containing complexing and } \\
\text { buffering agents }\end{array}$ & $\begin{array}{c}\text { Particulate fouling, metal } \\
\text { hydroxides, calcium carbonate }\end{array}$ \\
\hline \multirow{4}{*}{ Avista } & $\begin{array}{l}\text { RoClean } \\
\text { L403 }\end{array}$ & $\begin{array}{l}\text { A multicomponent and low } \mathrm{pH} \\
\text { cleaner }\end{array}$ & $\begin{array}{l}\text { All acid removable scaling and } \\
\text { foulants }\end{array}$ \\
\hline & $\begin{array}{l}\text { RoClean } \\
\text { L211 }\end{array}$ & $\begin{array}{l}\text { A multicomponent, high } \mathrm{pH} \\
\text { buffered, low foaming liquid } \\
\text { cleaner }\end{array}$ & $\begin{array}{l}\text { Colloidal silica, clay, organic } \\
\text { color and bioslime }\end{array}$ \\
\hline & $\begin{array}{l}\text { RoClean } \\
\text { L212 }\end{array}$ & $\begin{array}{l}\text { An ethylenediaminetetraacetic acid } \\
\text { (EDTA) and phosphate free liquid } \\
\text { cleaner with a pH of } 11.7-12.7\end{array}$ & $\begin{array}{l}\text { Colloidal silica, clays, organic } \\
\text { color and bioslime }\end{array}$ \\
\hline & $\begin{array}{l}\text { RoClean } \\
\text { P112 }\end{array}$ & $\begin{array}{l}\text { A multicomponent cleaner with a } \\
\qquad \mathrm{pH} \text { of } 12.0-12.9\end{array}$ & Silica scaling \\
\hline \multirow{3}{*}{ AWC } & C-234 & $\begin{array}{l}\text { A low } \mathrm{pH} \text { cleaner including a } \\
\text { mixture of cleaners and scaling } \\
\text { removal agents }\end{array}$ & $\begin{array}{l}\text { Inorganic carbonate, phosphate } \\
\text { and sulfate scaling as well as } \\
\text { metal oxides }\end{array}$ \\
\hline & $\mathrm{C}-235$ & $\begin{array}{l}\text { A low pH phosphate-free cleaner } \\
\text { including a mixture of cleaners, } \\
\text { chelants and descaling agents }\end{array}$ & $\begin{array}{c}\text { Inorganic carbonate and } \\
\text { phosphate scaling as well as metal } \\
\text { oxide/hydroxide foulants }\end{array}$ \\
\hline & $\mathrm{C}-236$ & $\begin{array}{l}\text { A high pH silica cleaner with } \\
\text { chelating agents }\end{array}$ & $\begin{array}{l}\text { Polymerized silica scaling as well } \\
\text { as colloidal silica fouling }\end{array}$ \\
\hline \multicolumn{4}{|c|}{ Commercial cleaning agents designed for organic fouling } \\
\hline \multirow{2}{*}{ Ecolab } & Ultrasil $67^{1}$ & $\begin{array}{c}\text { A neutral } \mathrm{pH}, \text { surfactant-based } \\
\text { cleaner with enzymes }\end{array}$ & Proteins \\
\hline & Ultrasil $69^{1}$ & $\begin{array}{l}\text { An alkaline cleaner with organic / } \\
\text { inorganic chelating agents }\end{array}$ & Organics \\
\hline
\end{tabular}




\begin{tabular}{|c|c|c|c|}
\hline & Ultrasil 110 & An alkaline liquid cleaner & $\begin{array}{l}\text { Proteins and other organic } \\
\text { residues }\end{array}$ \\
\hline & Ultrasil 112 & $\begin{array}{c}\text { An alkaline cleaner contains } 5 \% \\
\text { alkylbenzene sulfonate }\end{array}$ & Organics \\
\hline \multirow[t]{3}{*}{ BWA } & Flocon MC11 & $\begin{array}{l}\text { Mixture of detergent builders, } \\
\text { chelating agents and } \mathrm{pH} \text { buffer }\end{array}$ & $\begin{array}{l}\text { Organics, silt and other } \\
\text { particulates }\end{array}$ \\
\hline & $\begin{array}{c}\text { Kleen*MCT4 } \\
04\end{array}$ & $\begin{array}{l}\text { A neutral } \mathrm{pH} \text {, anionic surfactant- } \\
\text { based liquid formulation }\end{array}$ & $\begin{array}{l}\text { Organic matter, colloidal foulants } \\
\text { and silt }\end{array}$ \\
\hline & $\begin{array}{c}\text { Kleen*MCT5 } \\
11\end{array}$ & $\begin{array}{l}\text { A high } \mathrm{pH} \text { cleaner containing a } \\
\text { mixture of solubilizing and } \\
\text { complexing agents }\end{array}$ & $\begin{array}{l}\text { Organics, silt, and other } \\
\text { particulates }\end{array}$ \\
\hline \multirow{3}{*}{ Suez (GE) } & $\begin{array}{c}\text { Kleen*MCT5 } \\
24\end{array}$ & $\begin{array}{l}\text { A high } \mathrm{pH} \text { cleaner containing a } \\
\text { mixture of solubilizing and } \\
\text { complexing agents }\end{array}$ & $\begin{array}{l}\text { Organics, silt, microbiological } \\
\text { metabolites and other particulates }\end{array}$ \\
\hline & $\begin{array}{c}\text { Kleen*MCT8 } \\
82\end{array}$ & $\begin{array}{l}\text { A low pH cleaner containing a } \\
\text { mixture of solubilizing and } \\
\text { complexing agents as well as a } \\
\text { surfactant }\end{array}$ & $\begin{array}{c}\text { Organic foulants (including } \\
\text { biological molecules) and scaling }\end{array}$ \\
\hline & $\begin{array}{l}\text { MemChem* } \\
\text { MCT505 }\end{array}$ & $\begin{array}{l}\text { A mildly alkaline liquid cleaner } \\
\text { containing nonylphenol ethoxylates } \\
\text { (NPE)-free surfactants, detergents, } \\
\text { and chelating agents }\end{array}$ & $\begin{array}{c}\text { Organics, greases, oils, oxides and } \\
\text { hard-water stains }\end{array}$ \\
\hline \multicolumn{4}{|c|}{ Commercial cleaning agents designed for biofouling } \\
\hline Ecolab & Oxonia active & $\begin{array}{l}\text { An acidic biocide containing } \\
\text { hydrogen peroxide / peracetic acid }\end{array}$ & All types of microorganisms. \\
\hline \multirow[t]{2}{*}{ Avista } & RoCide $^{\circledR}$ DB5 & $\begin{array}{c}\text { A } 5 \% \text { solution of the active } \\
\text { ingredient 2,2-dibromo-3- } \\
\text { nitrilopropionamide (DBNPA) with } \\
\text { a pH of } 1.5-5.0\end{array}$ & Microorganisms \\
\hline & $\begin{array}{c}\text { RoCide }^{\circledR} \\
\text { DB20 }\end{array}$ & $\begin{array}{c}\text { A } 20 \% \text { solution of the active } \\
\text { ingredient DBNPA with a pH of } \\
2.0-5.0\end{array}$ & Microorganisms \\
\hline Suez (GE) & $\begin{array}{l}\text { BioMate*MB } \\
\text { C781 }\end{array}$ & A non-oxidizing biocide & $\begin{array}{l}\text { Mitigation of bacterial growth and } \\
\text { slime build-up }\end{array}$ \\
\hline
\end{tabular}


1. Houari, A.; Di Martino, P., Polysaccharide-hydrolysing enzymes enhance the in vitro cleaning efficiency of Nanofiltration membranes. AIMS Microbiol. 2019, 5(4), 368. 